\title{
Treatment of chronic osteomyelitis with locally made calcium sulfate bone cement pellets impregnated with antibiotics at University Teaching Hospital of Butare (CHUB), Rwanda
}

\author{
Julien Gashegu ${ }^{1,2}$, Tom Byamungu ${ }^{2}$, Christian Ngarambe², Justin Bayisenga², Ahmed Kiswezi ${ }^{2}$ \\ 1. Clinical Anatomy Unit, School of Medicine and Pharmacy, University of Rwanda, Kigali, Rwanda \\ 2. Department of Surgery, University Teaching Hospital of Butare (CHUB), Butare, Rwanda \\ Correspondence: Prof. Julien Gashegu (kgashegu@gmail.com)
}

\begin{abstract}
(c) $2018 \mathrm{~J}$. Gashegu et al. This open access article is licensed under a Creative Commons Attribution 4.0 International License (http://creativecommons.org/licenses/by/4.0/), which permits unrestricted use, distribution, and reproduction in any medium, provided you give appropriate credit to the original author(s) and the source, provide a link to the Creative Commons license, and indicate if changes were made.
\end{abstract}

\begin{abstract}
Background

Chronic osteomyelitis remains a challenging and potentially lifelong disease. Its treatment includes surgery, which is essential and consists of the removal of all dead bone and necrotic soft tissues, and systemic antibiotic administration. One excellent modality for antibiotic delivery is the use of antibiotic-impregnated bone cement. In this paper, we report our experience of operating-room-made antibiotic-impregnated bone cement at University Teaching Hospital of Butare (CHUB), Rwanda.
\end{abstract}

\begin{abstract}
Methods
Fifty-five patients with haematogenously spread chronic osteomyelitis were operated upon and benefited from our protocol between January 2015 and December 2016. Excluded from this study were cases in which we estimated that soft tissue bone coverage might have been a challenge. We mixed $300 \mathrm{~g}$ of calcium sulfate powder with $2 \mathrm{~g}$ of ceftriaxone and $480 \mathrm{mg}$ of gentamicin to form pellets. To allow for drying, we prepared the mixture 2 hours before implantation into operated bone.
\end{abstract}

\begin{abstract}
Results
Thirty-one patients (56\%) were children under 15 years of age, and 44 (80\%) of the cases involved the tibia or the femur. After 12 months of follow-up, only 3 cases (5\%) presented with complications that needed additional surgeries. All complications were related to the biodegradation of the calcium sulfate pallets. The rest of the patients in this study improved without complications,
\end{abstract}

\section{Conclusions}

The locally made (within in the operating room) antibiotic-impregnated bone cement at CHUB was safe, effective, and of low cost.

Keywords: chronic osteomyelitis, bone cement, calcium sulfate, antibiotics, locally available materials, Rwanda

\section{Introduction}

Chronic osteomyelitis (COM) of the long bones is a common and debilitating condition in developing countries, particularly in Africa. Its management remains a challenge, and it is often a disease of the poor, commonly associated with malnutrition and compromised immunity.

In Rwanda, patients with COM often present late due to cultural beliefs (in Kinyarwanda COM is inzibyi and believed to be curable by traditional healers). Other challenges contributing to late presentation include economic issues, inaccessible modern health care, lack of proper information from the local communities, and failure of diagnosis of acute osteomyelitis by health professionals at primary health centres.

The key component of COM treatment is surgery to remove the dead bone, necrotic tissues, and purulent material from the medullary cavity of the bone.

The classical recommendation of postoperative antibiotic therapy for 4 to 6 weeks has poor compliance in our setting. Even the modified 3-week antibiotic course is often not fully complied with, for cultural and socioeconomic reasons. Furthermore, there is a logical scientific question regarding 
Table 1. Demographic characteristics

\begin{tabular}{c|c}
\hline Age (years) & n (\%) \\
\hline $0-15$ & $31(56)$ \\
$16-30$ & $18(33 \%)$ \\
$>30$ & $6(11 \%)$ \\
\hline \multicolumn{1}{c|}{ Total } & $\mathbf{5 5}$ \\
\hline Table 2. Affected bones & $\mathbf{n ~ ( \% )}$ \\
\hline Bone & $25(45 \%)$ \\
\hline Tibia & $19(35 \%)$ \\
Femur & $5(9 \%)$ \\
Calcaneus & $4(7 \%)$ \\
Humerus & $1(2 \%)$ \\
Clavicle & $1(2 \%)$ \\
\hline Ulna & $\mathbf{5 5}$ \\
\hline
\end{tabular}

the efficacy of long-course systemic antibiotic therapy, as the antibiotics hardly penetrate the COM bone site. There is no published scientific evidence supporting the efficacy of the long-course of antibiotic therapy. ${ }^{1}$

Calcium sulfate plaster of Paris powder has been demonstrated in experimental and clinical studies to be a suitable, biocompatible, and bioresorbable drug carrier. ${ }^{1}$ Antibiotic-impregnated calcium sulfate beads have been demonstrated to provide an adequate local antibiotic concentration at the site of COM for 2 to 3 weeks. ${ }^{2}$

In this paper, we present the experience of locally made antibiotic-impregnated calcium sulfate pellets as local antibiotic therapy for COM at University Teaching Hospital of Butare (CHUB), Rwanda.

\section{Methods}

\section{Locally made antibiotic-impregnated calcium sulfate pellets}

Calcium sulfate powder was packed in small packs of $300 \mathrm{~g}$ and sterilized for use within the next 5 days. In the operating room, the calcium sulfate was mixed with diluted antibiotics. A sterile pack of $300 \mathrm{~g}$ of calcium sulfate was mixed with 2 $\mathrm{g}$ of ceftriaxone and $480 \mathrm{mg}$ of gentamycin. The quantity of powder used, and by consequence, the quantity of antibiotic depended on the estimated size of the bone defect after removal of the sequestrum. We realized that it takes around 1 to 2 hours for the pellets to be dry enough to be inserted into the bone defect. For this reason, the bone cement pellets were made before the preparation of the operating sites to allow enough time for the cement to dry (Figure 1). After surgery, all patients received similar parenteral antibiotics for 5 days.

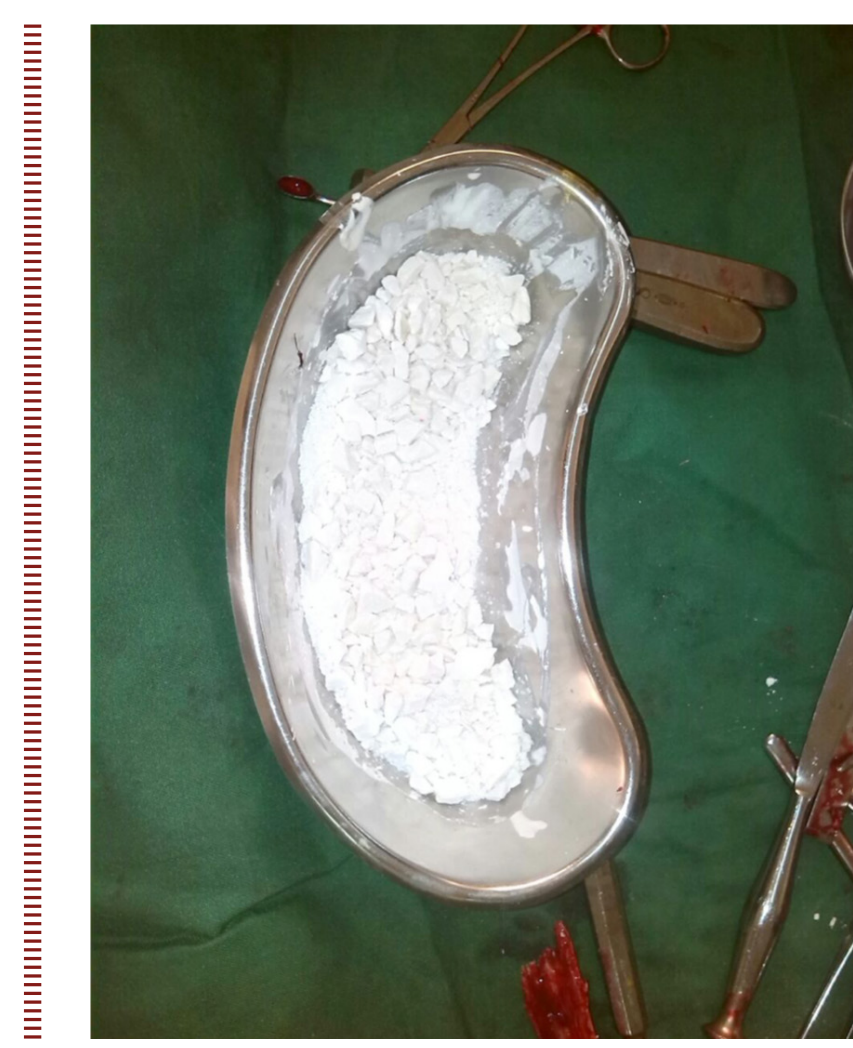

Figure 1. Operating room-made calcium sulfate bone cement antibiotic-impregnated pellets

\section{Surgical procedure}

The patients were operated on after receiving a definite diagnosis and providing written informed consent. The operations were carried out by 2 senior registrars with adequate experience in orthopaedic surgery. The surgical approaches depended on the affected bone. The affected bone was exposed, and generally, a canoe was made to access the medullary cavity (Figure 2).

The sequestrum was removed with all necrotic tissue in the cavity by generous bone curettage. After surgical toilet of the surgical site, the antibiotic-impregnated calcium sulfate pellets were inserted into the bone defect canoe (Figure 3 and Figure 4).

\section{Patients}

The patients who consulted our surgical department from January 2015 to December 2016, and had a diagnosis of COM, were candidates for our study. During this period, 65 patients underwent surgery for COM, 55 of whom were 


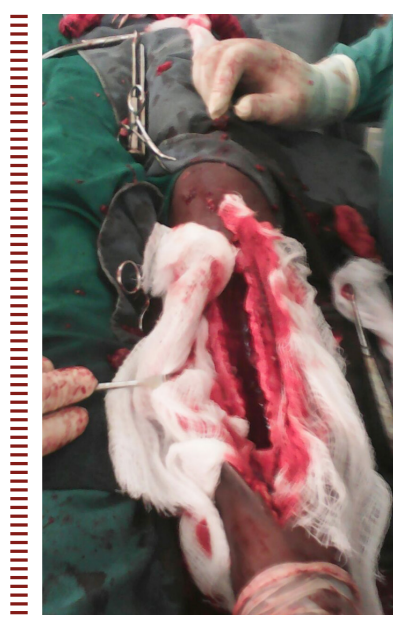

Figure 2. A canoe made in the tibia to access and remove the sequestrum and other necrotic tissues; this image shows the bone defect (canoe) after curettage.

enrolled in our study. We excluded patients with secondary osteomyelitis and those for whom we estimated that the soft tissue covering of the bone might be challenging. Data collection was cumulatively done using a standardized questionnaire for every participant, and analysis was done using Microsft Excel. After the procedure, there were 12 months of follow-up for all study patients.

\section{Ethical considerations}

Approval for this study was obtained from the Department of Surgery Ethics Committee, and the Institutional Review Board of the University Teaching Hospital of Butare. The data collected was kept confidential, and only used for this study. All patients provided written informed consent before participating in the study.

\section{Results}

From January 2015 to December 2016, 55 cases of COM were enrolled in this study and were followed up for 12 months. Table 1 shows the demographic characteristics of our patients and Table 2 shows the affected bones.

Of the 55 cases of COM treated with locally made bone cement, 3 cases (5\%) developed complications. Fifty-two cases (95\%) improved without any complications after 12 months of follow-up. Of the 3 cases with complications, 1 case of COM of the right femur developed an early foreign body rejection reaction against the bone cement; this patient underwent additional surgery to remove the bone cement pellets on the fifth postoperative day.

The other 2 complications presented late as incomplete absorption of the calcium sulfate-1 case of COM of the left femur and another of the right tibia. They developed serous discharge after 3 and 4 months, respectively, and the follow-up x-rays showed residual intramedullary calcium sulfate. The pellets were surgically removed.

\section{Discussion}

$\mathrm{COM}$ is a challenging and potentially lifelong condition. It is therefore essential to find strategies to eradicate the associated infectious organisms.

In this study, the majority of cases (56\%) were paediatric cases under 15 years of age, and most of them were from the rural and low-income families. Poor hygiene and poor nutrition were possibly among the risk factors this patient population.

Our study also showed that the tibia was the most affected bone, with $45 \%$ of the cases, and that the tibia and the femur represented $80 \%$ of all cases. These results are consistent with other studies. $^{3-5}$

In COM, the infected bone becomes devascularized and eventually sequestered as a portion of dead bone that contains bacterial colonies. ${ }^{6}$

Dead bone tissues with an insufficient blood supply interfere with normal healing and must be surgically removed. ${ }^{6}$

Appropriate antibiotic therapy supplements surgery. However, the site of infected bone often has impaired vasculature, and as a consequence, the antibiotic levels achieved following systemic administration may be much lower than expected, relative to the serum or the surrounding tissue levels. ${ }^{6}$

Intravenous or oral antibiotics for 4 to 6 weeks is a widely used protocol to treat COM. ${ }^{1}$ This duration of therapy remains empirical rather than evidence-based, as there are no documented records or publications indicating its superiority over other protocols. ${ }^{1}$

One good alternative for antibiotic therapy in COM is the use of a local antibiotic spacer. Antibiotic-impregnated bone cement was introduced in the 1970s to treat infected arthroplasties, and antibiotic-impregnated beads have since been developed to treat local soft tissue and bone infections. ${ }^{1,2}$ The advantage of antibiotic-containing beads is that they fill the dead space produced by sequestrectomy and bone curettage and the resulting local antibiotic concentrations vastly exceed the minimum inhibitory concentrations for most pathogens isolated in orthopaedic infections. ${ }^{1,2,6-12}$

Calcium sulfate can be loaded with antibiotics and used as a biodegradable medication delivery device. The advantage of calcium sulfate pellets is that they can offer the chance for delivery and sufficient concentrations of local antibiotics. With the biodegradation, they do not need to be surgically removed. ${ }^{2,6,8,11}$

Commercially manufactured bone cement is too expensive for the Rwandan population and probably for most of the African population. Additionally, it is not readily available in our market. In 2012, a manufacturing company from Europe tried to implement a bone cement protocol in Rwanda. Only 20 cases were successfully treated (unpublished data), and the protocol was stopped due to high costs that could not be met by our population. One set costs approximately US $\$ 200$, and some cases would need 4 to 5 sets of commercially manufactured bone cement to fill the bone defect after sequestrectomy. 

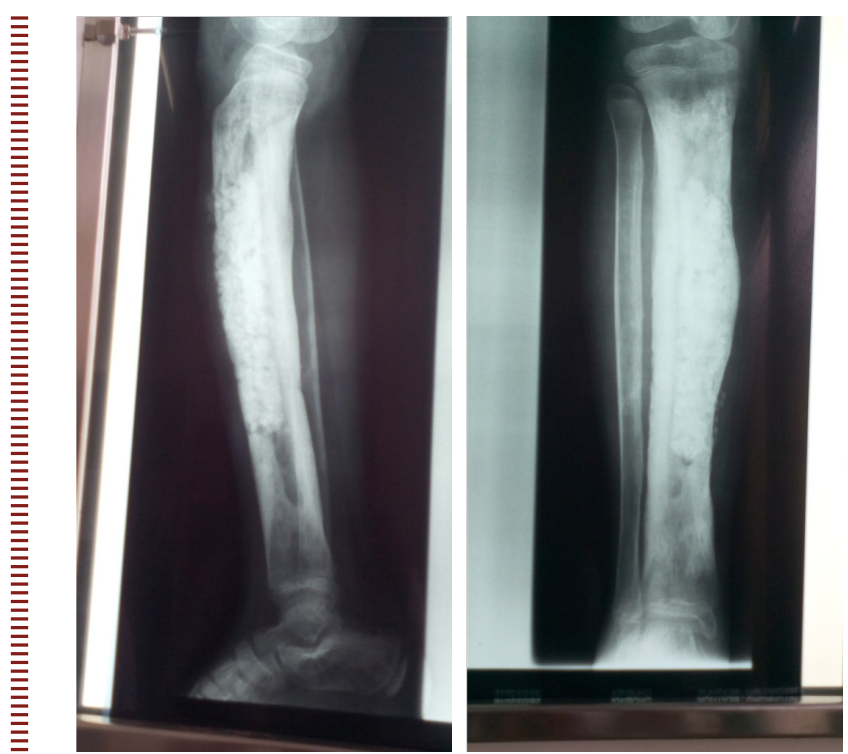

Figure 4. Postoperative tibia control x-ray images showing bone cement pellets filling the bone defect after sequestrectomy
Our experience, however, showed that calcium sulfate antibiotic-impregnated bone cement can be easily and safely made locally, providing a sound and sustainable option for localized antibiotic delivery in our setting.

There were, however, a few drawbacks. The degradation products of calcium sulfate carriers generally resulted in persistent drainage from the wound, and this may aggravate deep infections. Calcium sulfate absorbs plenty of water, which subsequently promotes seroma formation and increases the risk of secondary infection. ${ }^{2}$ Our experience also showed that the calcium sulfate might not be completely degraded, causing a foreign body reaction in a small number of cases.

\section{Conclusions}

Our study showed that COM is more frequent in paediatric patients with a risk of permanent sequelae. Locally made antibiotic-impregnated bone cement pellets were a safe, effective, and low-cost option for COM treatment in the Rwandan tertiary hospital setting.

\section{Competing interests}

All authors declare that they have no competing interests related to this work.

\section{References}

1. Haidar R, Der Boghossian A, Atiyeh B. Duration of post-surgical antibiotics in chronic osteomyelitis: empiric or evidence-based? Int J Infect Dis 2010 Sep;14(9):e752-8. doi: 10.1016/j. ijid.2010.01.005. Epub 2010 May 14.

2. Luo $S$, Jiang $T$, Yang $Y$, Yang $X$, Zhao J. Combination therapy with vancomycinloaded calcium sulfate and vancomycinloaded PMMA in the treatment of chronic osteomyelitis. BMC Musculoskelet Disord. 2016 Dec 22;17(1):502. doi: 10.1186/ s12891-016-1352-9.

3. Mantero E, Carbone M, Calevo MG, Boero S. Diagnosis and treatment of pediatric chronic osteomyelitis in developing countries: prospective study of 96 patients treated in Kenya. Musculoskelet Surg. 2011 Apr;95(1):13-8. doi: 10.1007/ s12306-011-0104-0. Epub 2011 Mar 5.

4. Marais LC, Ferreira N, Aldous C, Le Roux $T L$. The outcome of treatment of chronic osteomyelitis according to an integrated approach. Strategies Trauma Limb Reconstr. 2016 Aug;11(2):135-42. doi: 10.1007/s11751-016-0259-1. Epub 2016 Jul 1.

5. Museru LM, Mcharo CN. Chronic osteomyelitis: a continuing orthopaedic challenge in developing countries. Int Orthop. 2001:25(2):127-31. doi: 10.1007/ s002640100239.
6. Papagelopoulos PJ, Mavrogenis AF, Tsiodras S, Vlastou C, Giamarellou H Soucacos PN. Calcium sulphate delivery system with tobramycin for the treatment of chronic calcaneal osteomyelitis. J Int Med Res. 2006 Nov-Dec:34(6):704-12 do: $10.1177 / 147323000603400618$

7. Neut $D$, de Groot EP, Kowalski RS, van Horn JR, van der Mei HC, Busscher HJ. Gentamicin-loaded bone cement with clindamycin or fusidic acid added: biofilm formation and antibiotic release. J Biomed Mater Res A. 2005 May 1;73(2):165-70. doi: 10.1002/jbm.a.30253.

8. van Vugt TA, Geurts J, Arts JJ. Clinical application of antimicrobial bone graft substitute in osteomyelitis treatment: a systematic review of different bone graft substitutes available in clinica treatment of osteomyelitis. Biomed Res Int. 2016;2016:6984656. doi: 10.1155/2016/6984656. Epub 2016 Jan 21.

9. Thomas MV, Puleo DA, Al-Sabbagh M Calcium sulfate: a review. J Long Term Eff Med Implants. 2005:15(6):599-607.
10. Beardmore AA, Brooks DE, Wenke JC, Thomas DB. Effectiveness of local antibiotic delivery with an osteoinductive and osteoconductive bone-graft substitute. 2005 Jan;87(1):107-12. doi: 10.2106/JBJS.C. 01670 .

11. Thomas DB, Brooks DE, Bice TG, DeJong ES, Lonergan KT, Wenke JC. Tobramycinimpregnated calcium sulfate prevents infection in contaminated wounds. Clin Orthop Relat Res. 2005 Dec;441:366-71.

12. Humm G, Noor S, Bridgeman P, David M, Bose D. Adjuvant treatment of chronic osteomyelitis of the tibia following exogenous trauma using OSTEOSET( $\left.{ }^{\oplus}\right)-\mathrm{T}$ : a review of 21 patients in a regional trauma centre. Strategies Trauma Limb Reconstr. 2014 Nov;9(3):157-61. doi: 10.1007/s11751-014-0206-y. Epub 2014 Dec 25. 